\section{ECCOMAS}

Proceedia
COMPDYN 2021

$8^{\text {th }}$ ECCOMAS Thematic Conference on Computational Methods in Structural Dynamics and Earthquake Engineering

M. Papadrakakis, M. Fragiadakis (eds.) Streamed from Athens, Greece, 28 - 30 June 2021

\title{
PERFORMANCE-BASED EARTHQUAKE ENGINEERING ANALYSIS OF SHORT-MEDIUM SPAN STEEL-CONCRETE COMPOSITE BRIDGES
}

\author{
Daniele Corritore $^{1}$, Fabrizio Paolacci ${ }^{1}$ \\ ${ }^{1}$ Department of Engineering, Roma Tre University \\ e-mail: fabrizio.paolacci@uniroma3.it,daniele.corritore@uniroma3.it
}

\begin{abstract}
In this paper, the results of the performance-based earthquake engineering (PBEE) analysis, carried out to assess the seismic behaviour of short-medium span steel-concrete composite Igirder bridges, are presented and discussed. The selected case study is part of a group of bridges analysed within the SEQBRI project, funded in 2012 by the European Union, which deals with a systematic development of the PBEE analysis for short-medium span steel-concrete compo-site I-girder bridges. In this respect, fragility and damage analysis of the selected bridge are performed using a proper component-based numerical model along with wide experimental campaign. These outcomes are then integrated in the decision-making analysis, where the selected decision variable is the repair cost ratio of the bridge. The results show the good performances of short medium span steel-concrete composite bridges, both for minor and major damage scenarios.
\end{abstract}

Keywords: Performance based earthquake engineering, steel-concrete composite bridges, loss analysis, concrete cross beam.

ISSN:2623-3347 (C) 2021 The Authors. Published by Eccomas Proceedia.

Peer-review under responsibility of the organizing committee of COMPDYN 2021.

doi: $10.7712 / 120121.8721 .18783$ 


\section{INTRODUCTION}

Nowadays, short-medium span steel-concrete composite I-girder bridges (SCC) made of hot rolled steel beams are very popular, owing to their short construction time and reduced costs. Moreover, they are very adequate for seismic areas for their limited weight. These bridges can be easily designed for static loading, according to Eurocode 4, [1]; however, the effects of seismic loading has not been yet adequately investigated, and thus, they may exhibit damages even in low-seismicity zones.

In general, steel-concrete composite bridges with hot rolled sections for small and medium spans, (range between 25-40 m), exhibit several advantages in terms of: small total depth of composite section, no pre-stressing in concrete slab, low dead weight and limited foundations and settlements of supports, simple erection methods. The SEQBRI project, funded in 2012 by the European Union and conceived on these premises, deals with a systematic development of the Performance-Based Earthquake Engineering, [2], for short-medium span steel-concrete composite I-girder bridges that includes seismic input randomness, demand and damage analysis as well as economic cost-benefit estimations, [3]. In particular, a new type of connection between steel girder and piers that use a transverse concrete cross beam (CCB) is taken into account. The steelwork parts of the composite beams are prepared in factory and equipped with steel elements for connections (plates, holes, welded studs...). The steel girders are placed on the head of the piers without any propping; subsequently, the slab is concreted and the continuity of the composite beam is completed by concreting the beam joints and the transverse beams over the pier. This kind of joint has been studied in the past but only for static load conditions, [4-5]. In this paper, the results of the performance-based earthquake engineering (PBEE) analysis, carried out to assess the seismic performance of short-medium span steel-concrete composite I-girder bridges, are presented and dis-cussed. The selected case study is part of a group of bridges analysed within the SEQBRI project, [3]. In this respect, fragility and damage analysis of the selected bridge are performed based on a proper component-based numerical model and a wide experimental campaign, [6-7]. These outcomes are then integrated in the decision making analysis, where the selected decision variable is the repair cost ratio of the bridge.

\section{DESCRIPTION OF THE CASE STUDY}

The analyzed case study is a straight 2-span deck consisting of 4 main girders HE600B of S460 steel grade with $2.65 \mathrm{~m}$ in-between distance (Figure1). The bridge is $40.00 \mathrm{~m}$ long and consists of 2 spans; the total width of the road cross-section is $10.60 \mathrm{~m}$, with carriageway $6.50 \mathrm{~m}$ wide and 2 sidewalks $2.05 \mathrm{~m}$ wide. The thickness of the concrete slab is equal to $25 \mathrm{~cm}$. At the abutments, the steel girders are fixed to an end reinforced concrete crossbeam $0.60 \mathrm{~m}$ wide. The deck is simply supported on normal damping rubber bearings.

At the intermediate pier, the steel girders are fixed to an intermediate reinforced concrete crossbeam $0.90 \mathrm{~m}$ wide (Figure2). A wall type pier $0.60 \mathrm{~m}$ thick and $7.00 \mathrm{~m}$ wide is used. It is assumed that the foundation soil is categorized as type B according to EN1998. In this example, the SSI effect is neglected.

Different types of CCB configurations have been applied in several occasions, [8]. Many of them are variants of the configurations included in the standard DIN-FB-104, [9]. These configurations are proposed in order to manage mainly vertical dead and live loads, which produce negative moments at supports. For bridges subjected to seismic actions, significant tensile forces might be exhibited also at the bottom flange of the steel girder, especially when monolithic connection between CCB and pier is formed. Because of the CCB configuration is the most crucial detail, in order to identify the better solution for applicability in seismic prone areas, within the SEQBRI project the DIN FB variant C of the CCB has been chosen to be 
analytically and experimentally investigated. Moreover, two new variants, based on the DIN FB variant $B$, have been designed and investigated.

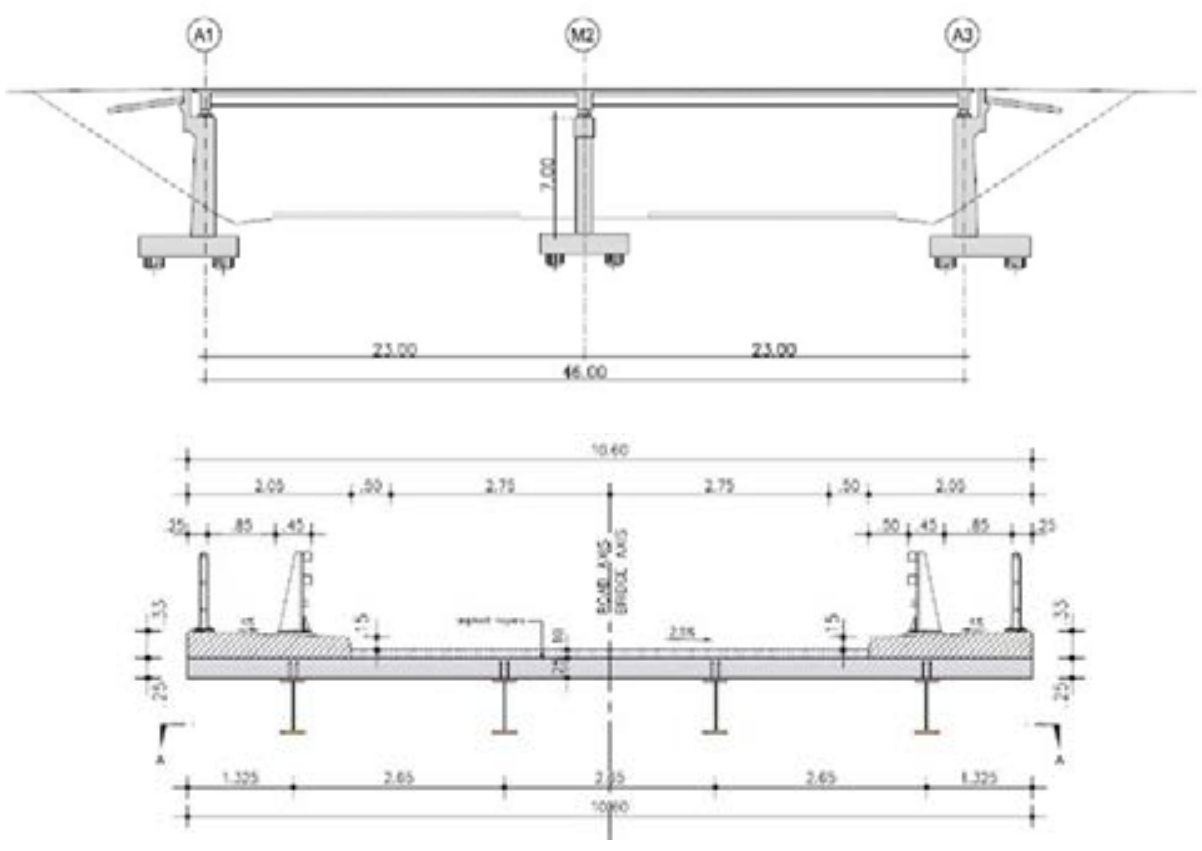

Figure 1. Longitudinal section and cross section at span for selected case study

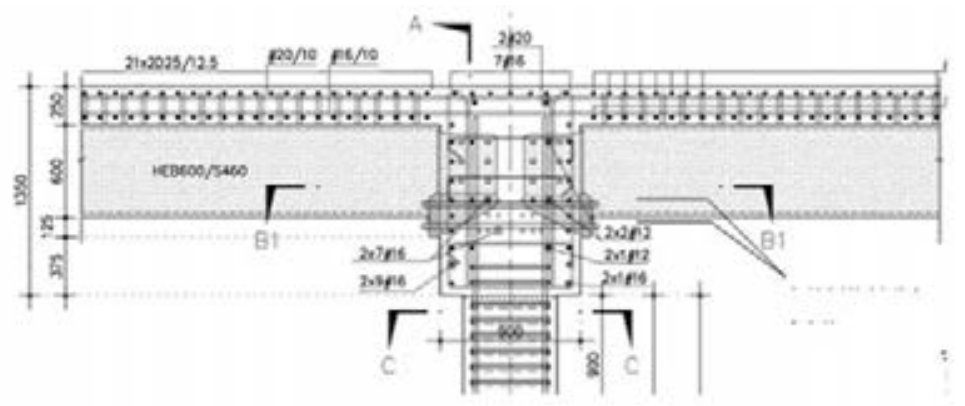

(a)

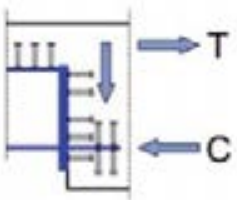

$\mathrm{CCB} 1$

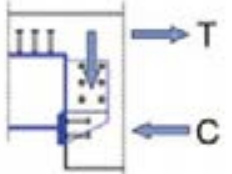

$\mathrm{CCB} 2$

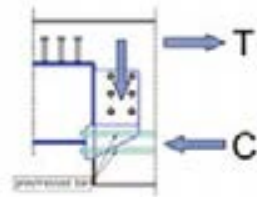

$\mathrm{CCB} 3$

(b)

Figure 2. (a) Pier-Deck joint with Concrete Cross Beam, (b) Static behavior of Pier-to-deck Joints

The idea is to transfer the forces from the composite girder to the CCB through contact and dedicated groups of shear studs. For this reason, in the new configurations, the steel flanges are not inserted into the CCB and the height of the head plate is limited (Figure 2). A broad 
description of these typologies and of the experimental texts can be found in Abbiati et al., [6] and Paolacci et al., [7]. In what follows the vulnerability of the selected bridge with CCB3 (see Figure 2b) is analyzed in the framework of PBEE. In this kind of CCB, the tensile force at the top flange is transferred gradually to the longitudinal reinforcement (or reversely a potential compression force to the concrete slab) through a group of vertical studs, which are placed on the top flange of the girder before the CCB. The shear force is transferred to the CCB through a group of horizontal studs, transversally placed at the sides of the girder's web, which is inserted into the CCB. These studs are subjected only to pure shear, action that is compatible to their actual function. The tensile stress at the bottom flange of the steel girder is transferred through anchor bars inserted throughout holes present in the web. The bars are tensioned after hardening of concrete and imposition of dead loads and are not bounded. The duct of each anchor should be filled with grease, in order to offer the opportunity for inspection, re-tension or even replacement in case of extreme seismic events.

\section{NUMERICAL MODELING OF CONCRETE CROSS BEAM -STEEL GIRDER CONNECTION}

A component based mechanical model of the CCB3 connection was developed in the code OpeSEES, [10]. This model (Figure 3) was used to analytically reproduce the experimental texts executed on I-girder subassemblies of the bridge. In [7] the results of the test campaign are presented and discussed. The aim was the definition of a refined model that faithfully approximates the real behavior of the sample and that allows the extension of the model to several cases.

Nonlinearities have been explicitly considered in the steel girders, concrete slab, pier, shear headed studs of the CCB, shear headed studs between girder and slab. Steel girder and concrete slab have been modeled using nonlinear beam elements with fiber sections. The steel girder and the slab have been connected by elastic beam elements with high stiffness; whereas, the shear and axial behavior of the vertical connecting studs has been modeled using nonlinear links with elasto-plastic behaviour.

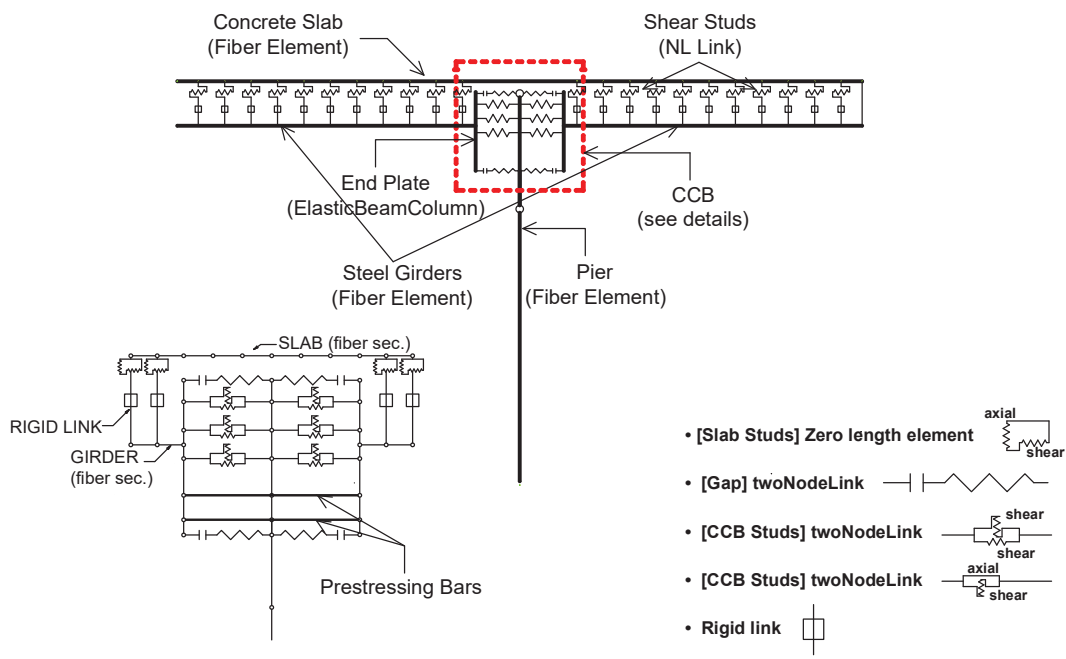

Figure 3. Numerical model of the pier-deck joint with Concrete Cross Beam 


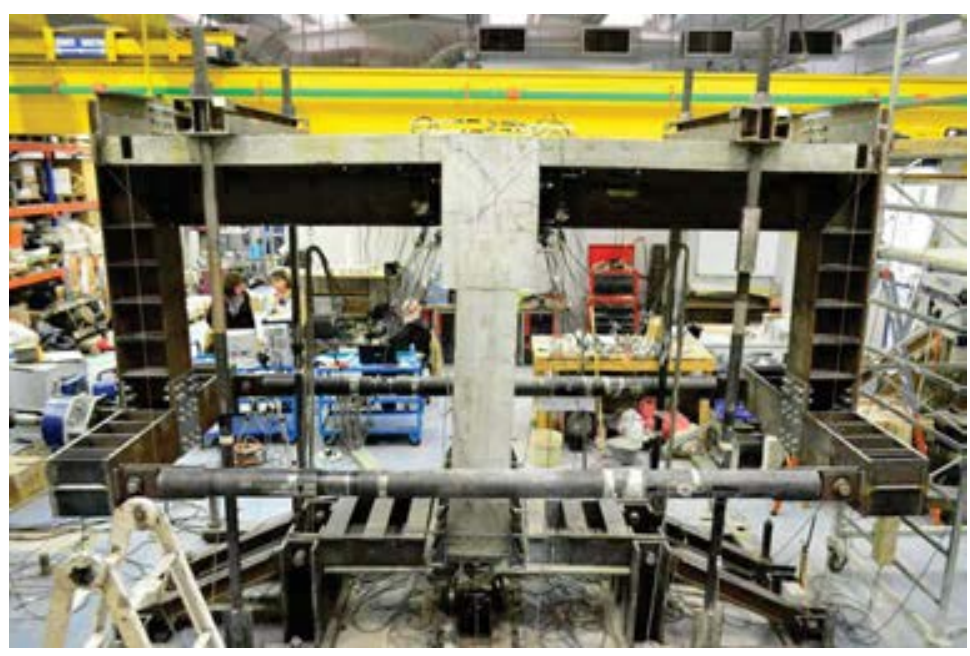

Fig. 4 - Experimental setup

Strength of the single shear stud $\mathrm{Q}_{\mathrm{u}}$ has been calculated according to CEN 1994, [1], while the related stiffness has been evaluated by mean of the shear load-slip curves (Q-s) defined in [11]:

$$
\frac{Q}{Q_{u}}=a \sqrt{1-e^{\frac{\beta_{s}}{a}}}+\gamma s
$$

with $\alpha=0.97, \beta=1.3, \gamma=0.0045 \mathrm{~mm}^{-1}$.

The vertical head plates welded on the steel girders and directly in contact with the transverse concrete beam have been modeled by rigid links. The CCB have been modeled by elastic beam elements with vertical axis. Nonlinearities in the CCB were considered by modeling shear studs and prestressing bars separately. Moreover, in order to reproduce the constraint in compression due to the presence of the concrete crossbeam, four compression gap elements have been used both at left and right sides of the CCB joints at two different levels, as shown in Figure 3, [12]. A simplified evaluation of the stiffness of the gap has been performed, assuming that the compressive force coming from the girder would act uniformly on a surface equal to the area of the vertical head-plate.

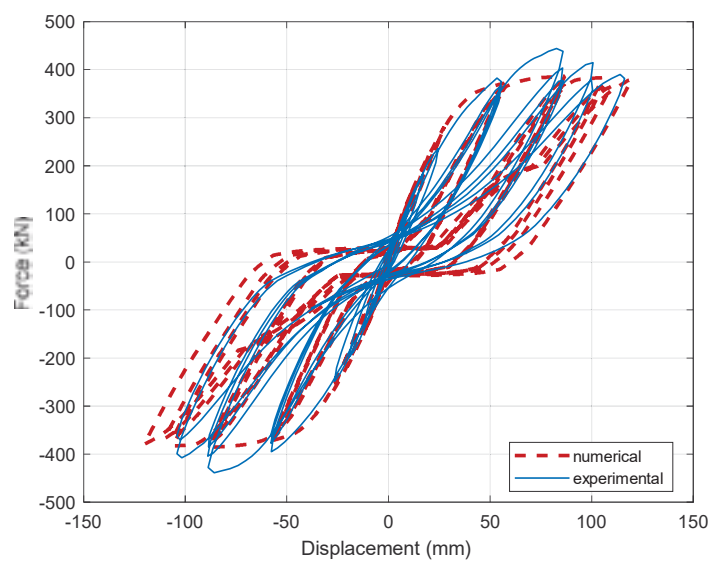

Figure 5. Numerical and experimental hysteretic response 
Studs welded in the transverse direction on the web plate entering the CCB are simulated with nonlinear links in the vertical and horizontal direction. Each links simulates the effect of $\phi 25$ $\mathrm{mm}$ shear studs. Their strength and stiffness has been calculated as for the studs in the slab. The two levels of pretension bars (Dywidag type) in the bottom area of the CCB, have been modeled by two elastic truss elements.

The 2-D FE model for concrete cross beam is shown in the following figure. This model has been validated through a wide experimental campaign. The setup of a test and a numerical/experimental comparison of the hysteretic force-displacement cycle are illustrated, respectively, in Figure 4 and in Figure 5.

\section{SESMIC INPUT SELECTION}

A group of 14 accelerograms has been selected from the PEER ground motion database in order to best approximate the Eurocode8 type 1 Response Spectrum, [13]. A set of records have been picked out from the database according to the following hazard parameters: magnitude range $\mathrm{Mw}=5.8-7.0$, distance from the fault rupture comprised in a range $15-40 \mathrm{~km}$ and local soil type B. Successive-ly, 14 records have been selected among these so that their mean spectrum best approximate the target spectrum. A scaling factor has been applied to each input signal in order to minimize the dispersion of the mean spectra compared to the Eurocode spetrum. The scaling factor modifies the original record only in amplitude.

\section{PROBABILISTIC SEISMIC DEMAND ANALYSIS OF CASE STUDIES}

The author's name should include first name, middle initial and surname. It should be written centered, in 12pt boldface Roman, 12pt below the title.

In the perspective of a Cost-Effective Risk Analysis, as the final goal of the application of the PBEE procedure, the elements (structural or finishing) whose damage and repairing item could be significant must be identified. Accordingly, the damage mechanisms and the Engineering Demand Parmeters (EDP) through which they can be quantifiyed by a numerical structural analysis must be defined. The selected EDPs for the chosen representative case study are listed hereafter, according to the classification of Mackie and Stojadinovic [14], and Tubaldi et al. [15]:

1. Global EDPs:

- $\quad$ Pier drift top displacement of the pier normalized with respect to the total height.

2. Intermediate EDPs

- $\quad$ Bending moment-curvature $\left(\mathrm{M}_{\max ,} \chi_{\max \square}\right)$ of the pier at the bottom section.

- Bending moment-rotation of the $\mathrm{CCB}$ connection.

3. Local EDPs

- Maximum force and relative displacement of shear studs in the CCB in the horizontal direction.

The bending moment and the curvature of the $\mathrm{CCB}$ connection have been obtained for both the left and right sides of the joint. The bending moment of the composite section just before the side of interest, has been defined as follows:

$$
M_{\text {max }}=M_{\text {slab }}+M_{\text {girder }}+\Delta N \cdot z
$$


where $M_{\text {slab }}$ is Mslabthe bending moment of the slab, $M_{\text {girder }}$ Mgirder the bending moment of the girder, $\Delta N$ the axial force in the slab and $z$ the level arm (distance between girder and slab). The rotation of one side of the joint is given by the rotation of the vertical end plate with respect to the undeformed configuration.

Incremental dynamic analyses have been performed, [16], and multi-record IDA curves of the structural response quantities mentioned above have been built. Since the properties of the seismic response depend on the intensity of the ground shaking, a comprehensive assessment requires numerous nonlinear dynamic analyses at various levels of intensity in order to represent different possible earthquake scenarios. The selected scan of PGA is in the range $(0.1 \mathrm{~g}-2.0 \mathrm{~g})$. The multi-record IDA response of the pier drift, obtained as mean value of the multi-record IDA, is shown in Figure 7. The mean value of the drift ratio for a PGA of $2.0 \mathrm{~g}$ is about $7 \%$. The typical collapse ratio of $4 \%$, [17], is reached around $1.2 \mathrm{~g}$ of PGA. For a PGA of $0.3 \mathrm{~g}$, which corresponds to the PGA of response spectrum type 1 (soil B) chosen for the design of the bridge, the drift ratio is about $1.0 \%$. Analyzing the moment-curvature relationship, not shown here for brevity, it can be noticed the nonlinear behaviour of the section at the base of the pier.

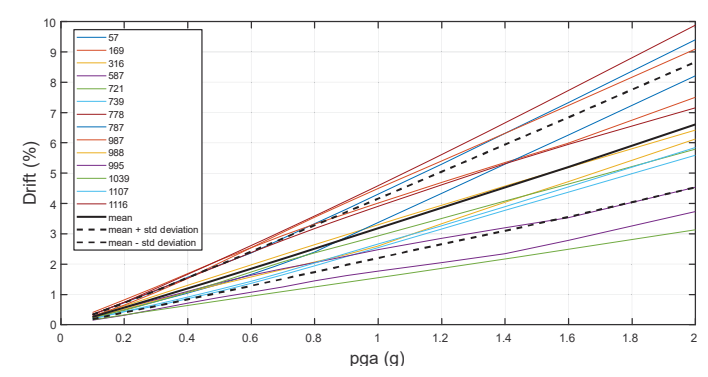

Figure 7. IDA-Drift ratio of the pier

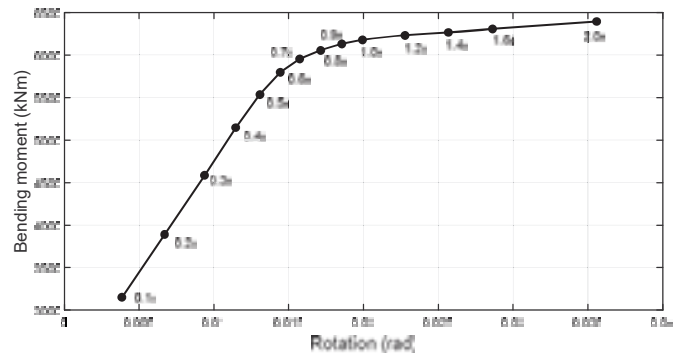

Figure 8. Bending moment-Rotation (mean values) of CCB left side

Pier yields at its base for earthquakes characterized by PGA of $0.4 \mathrm{~g}$ while plastic hinge develops for stronger seismic actions. Ultimate value is obtained between $1.2 \mathrm{~g}$ and $1.4 \mathrm{~g}$.

In Figure 8 the bending moment-rotation relationship (mean value) of CCB at left side, that is the maximum absolute bending moment versus the corresponding rotation of left side of the joint, is shown. It can be noticed that the onset of nonlinear behaviour takes place "after" the yielding of the pier, or rather for higher values of PGA (0.8-0.9 g). Representative force-slip curves of the CCB shear studs, not shown here for brevity, showed that, when the lower group begins to yield, around $0.5 \mathrm{~g}$, the upper levels of connectors are subject to smaller levels of stress. In particular, 1st group is still in the elastic range. The bigger deformations of the last line of studs are in agreement with the fact that during the seismic action the neutral axis in the CCB is closer to the first rows of connectors. In conclusion, for events with a PGA of $0.3 \mathrm{~g}$ or under, the critical part is the pier only with the formation of a plastic hinge at its base while the concrete crossbeam does not seem to be affected by any relevant damage. First level of damage within the intermediate CCB starts to arise from 0.5-0.6g, value of PGA for which the lower group of studs within the CCB yield.

\section{DAMAGE ANALYSIS AND FRAGILITY CURVES}

In order to implement performance-based earthquake engineering method for the representative case of small-medium span composite bridge and investigate its vulnerability, it has been necessary to accurately assess the probability of reaching particular levels of damage. For this purpose, a correlation process between the most representative engineering demand parameters and damage measures has been made. Piers are often the most vulnerable elements in a bridge 
and column failure can have catastrophic consequences. Excessive deformations can result in spalling of cover concrete, buckling of longitudinal reinforcement or bar fracture. Concrete cover spalling represents the first flexural damage state, in which there are marginal safety implications, there may be a possible short-term loss of function, and the cost to repair concrete spalling could be significant. Buckling and fracture of the longitudinal bars represent damage states in which safety implications are significant, partial replacement may be required, and a longer term loss of function may occur. In order to quantitatively implement PBEE for the considered reinforced wall-type pier, in this work the three damage conditions above mentioned have been considered as damage measures. The equations proposed by Berry and Eberhard, [18], for damage estimation have been used to relate the pier EDP and DS. Assuming that the DS occur in sequence (with DS1 preceding DS2 which, in turn, precedes DS3 and so on), the corresponding EDP can be ordered $\left(E D P_{1} \leq E D P_{2} \leq E D P_{3} \leq \cdots\right)$. Assuming, as an example, three DS, the joint probability density function of the variables $E D P_{1} \leq E D P_{2} \leq E D P_{3}$ can be assumed as follows:

$p_{E D P_{1}, E D P_{2}, E D P_{3}}\left(E D P_{1}, E D P_{2}, E D P_{3}\right)=\left\{\begin{array}{lr}\frac{1}{k} p_{L}\left(E D P_{1}, E D P_{2}, E D P_{3}\right) & \text { if } E D P_{1}, E D P_{2}, E D P_{3} \\ 0 & \text { otherwise }\end{array}\right.$

where $p_{E D P_{1}, E D P_{2}, E D P_{3}}($.$) is a joint lognormal distribution function of three random variables.$ The constant $k$ in the previous equation is a normalization constant. A more detailed explanation of the previous equation can be found in Alessandri et al., [19]. Figure 9 shows the four probabilities of the DS conditioned to the EDP evaluated for the pier. The correlation between the DS has been considered and the Damage States were assumed to occur in sequence (with concrete spalling preceding bar buckling, which, in turn, precedes bar fracture). For the shear headed studs of the beam-to-beam concrete connection, two levels of damage have been considered. In particular, first level of damage is the yielding of the studs, assumed as the last point of their elastic behaviour in the elasto-plastic constitutive law chosen in the model. The second level of damage corresponds to the complete rupture of the stud, taken from the research work of Gattesco and Giuriani, [11]. The rupture of a shear headed stud, monotonically loaded, takes place for a horizontal slip of $9.7 \mathrm{~mm}$. By assuming that the EDP is log-normally distributed, the probability of damage conditioned to the IM, $\mathrm{P}(\mathrm{DS} \mid \mathrm{IM})$, known as fragility function, is evaluated by the convolution of the $\mathrm{P}(\mathrm{DS} \mid \mathrm{EDP})$ and $\mathrm{P}(\mathrm{EDP} \mid \mathrm{IM})$ :

$$
\operatorname{Pr}(D S \mid \mathrm{im})=\int_{E D P} \operatorname{Pr}(D S \mid E D P) d G(E D P \mid I M) d E D P
$$

The DS0 in the figure corresponds to a negligible damage for which no repair items is considered. As shown in Figure 10, the probability of having cover spalling is over $15 \%$ for $0.3 \mathrm{~g}$ of PGA. It grows then very fast, reaching $80 \%$ at $0.75 \mathrm{~g}$, than reduces because of bar buckling and bar fracture become more probable. The probability of having bar buckling is under $10 \%$ until $0.8 \mathrm{~g}$ and reaches $30 \%$ at $2 \mathrm{~g}$. The risk of bar fracture seems to arise only for strongest seismic inputs. The probability of fracture of the reinforcement is under $10 \%$ until $0.8 \mathrm{~g}$, it is almost $30 \%$ for $1.6 \mathrm{~g}$ and reaches $35 \%$ for $2.0 \mathrm{~g}$. Figure 10 show the fragility function evaluated for the pier base. 


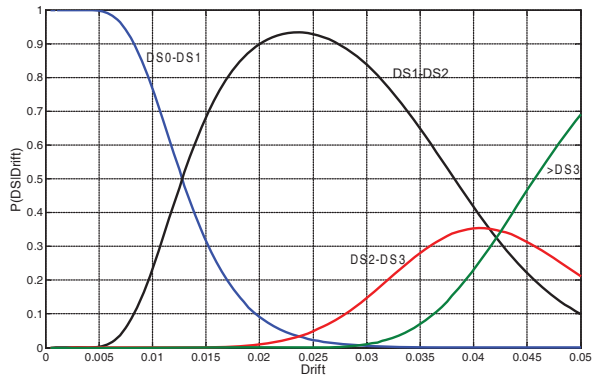

Figure 9. Conditional probability functions of the DS for the pier

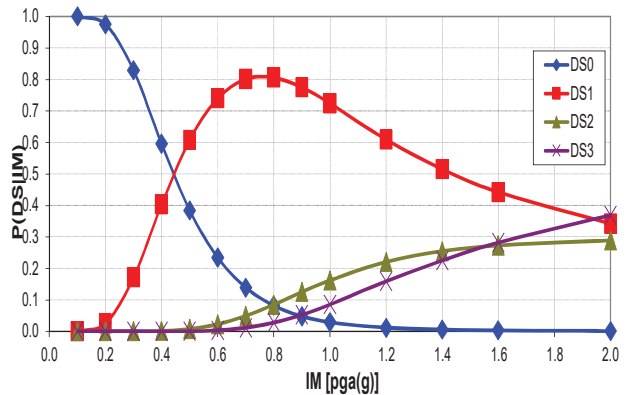

Figure 10. Fragility functions for the pier base

\section{COST-EFFECTIVE RISK ANALYSIS}

Cost-effective risk evaluation is the very last step of the global integrated probabilistic performance-based evaluation procedure used for SEQBRI project. The result of this phase is a decision model, which is based on Decision Variables (DV) pertaining to the use of a structure given a level of sustained damage. Decision models are mathematical relations between damage measures (DMs) and decision variables (DVs). Commonly used decision variables describe losses in terms of repair cost, interruption in service (downtime or repair time), or loss of life: thus, decision models are also called loss models. The decision model may have several parts those that relate DMs to repair quantities $(\mathrm{Q})$ and those that relate $\mathrm{Q}$ back to repair cost or repair time. A completely different set of decision variables focuses on the remaining capacity of the structure to function as intended. For example, the return of a highway bridge to differing degrees of functionality in a highway network is also an important loss criterion. Functionality may be measured in terms of lateral load resistance in aftershocks, traffic volume, lane and speed reductions, or access for emergency vehicles. The main DV here adopted is the repair/construction cost ratio (Direct cost). This choice comes from the goal to provide an immediate economic measure of earthquake performance of the assessed structure. Indirect costs like downtimes are not explicitly considered in the analysis. Global earthquake demand is defined in terms of peak ground acceleration (PGA), which represents the most commonly used intensity measure for structural seismic analysis and design issues. Local engineering demand parameters (EDP) consist in variables related to structural elements deformation or load, such as pier drift or bending moments. Damage states are expressed in terms of EDP limit values and associated damage or repair amounts expressed in terms of percentage of structural quantities (concrete cracking surface and/or volume, percentage of broken or yielded reinforcement rebars, percentage of broken studs or rotation angle for example for what concerns $\mathrm{CCB}$ ). At the end, the ultimate goal is to evaluate the cumulative probability distribution function of the decision variables DV (cost and/or repair duration), as expressed by the PBEE framework Integral defined by Equation (4):

$$
\lambda(D V<d v)=\int_{I M} \int_{D M} \int_{E D P} G(D V \mid D M) d G(D M \mid E D P) d G(E D P \mid I M)|d \lambda(I M)|
$$

where $\mathrm{G}(\cdot)$ represents the complementary cumulative distribution function of the considered variables.

Most of those different successive analysis parameters and intermediate outcomes, result from the previous tasks, including numerical non-linear analysis and experimental evaluations. At each steps, results are addressed in terms of probabilistic quantities such as mean values, 
deviation or probability of exceedance (see previous paragraphs). Repair costs and duration databases are collected from different former projects, US and French repair data, [3]. Additional (but rather sparse) data are also collected from road/bridges maintenance services concerning European recent « real field » seismic events such as Aquila earthquake. Analysis preferentially focuses on direct costs related to structural damages, since indirect costs appears to be very depending on the context (country, location, proximity of big cities, economical/industrial activities, toll-roads) and are not directly related to SEQBRI project bridge typology and specificity (SCC bridges with $\mathrm{CCB}$ ).

\subsection{Direct losses evaluation}

Direct cost evaluation is essentially based on repair quantities evaluated for different parts or elements of the structure for a given intensity measure or damage scenario and on associated unit repair costs. Repair cost estimation (and loss modeling in general) is almost always a structural system problem, not simply determined at the component level. Therefore, it is often necessary to combine (sum) numerous scalar values in order to obtain, for example, a total cost. For this matter, the bridge system is disaggregated into individual components or ensembles, such as the columns or CCB, denoted as performance groups (PGs) that are damaged, assessed, and repaired together using a specific combination of different repair methods. Each of those performance groups is also sub-divided into individual elements or sub-PGs.

\subsection{Performance Groups}

Different damage-states (DS) are defined for each performance group. The tested case-study is thus broken down into performance groups (PGs) for each major bridge component. Each performance group represents a collection of structural compo-nents that act as a global-level indicator of structural performance and that contribute significantly to re-pair-level decisions. Performance groups are not necessarily the same as load resisting structural components. For example, non-structural compo-nents may also be a performance group, since they also suffer damage and contribute to repair costs. The PGs generally correspond to things that are observable as a unit. Grouping bridge components into performance groups allows for more meaningful damage assessment than grouping by component. The definition of the PGs used for the SCC bridges with CCB corresponds to the following structure disaggregation. Four performance groups have been defined for this study (foundations are excluded):

- PG1: Pier (bottom)

- PG2: Pier (top)

- PG3: Concrete Cross beams

- PG4: Deck: including concrete slab, steel girders and superstructures/equipment.

\subsection{Damage States and associated repair methods and quantities}

Each damage state corresponds to only one repair method. Selection of repair methods is a crucial step in computing the repair-related decision variable, repair cost ratios and repair time. A selection of repair methods specific for the damage states has been made based on Caltrans database, [13], of typical repair techniques, since it has been defined to be generalizable and usable for performance-based evaluation of other structures. Each performance group contains a number of discrete damage states (DS) corresponding to repair quantities needed for restoring the bridge. The damage states are numbered from DS0 to DS3 with higher numbers indicating more severe damage. The DS0 damage state corresponds to the onset of damage when repair costs begin to accumulate. For analysis, the repair cost of the bridge is treated as $0 €$ below the 
DS0 level of damage. Damage beyond DS0 are needed to be repaired and costs begin to accumulate. Slight damage less than DS0 is assumed to be insignificant and not needing repair. Damage computation requires the definition of maximum possible repair quantities to define an upper limit to the quantities and costs. The upper limit is called DS3, since it corresponds to the most severe possible damage state for the elements in a performance group. DS3 usually corresponds to complete failure and replacement of all elements in the entire performance group. Details on repair methods here adopted can be found in [3].

\subsection{Damage Scenario and loss analysis}

To evaluate the expected repair cost of a structure as consequence of a seismic event in a given reference time period, one or more damage scenario must be defined. Its definition, which considers repairing items more or less large, reflects a level of the intensity measure (IM) of the ground motion at the site. Two damage scenarios have been defined; they are described in Table 1 and Table 2.

Table 1. Damage States (DS) for Minor scenario.

\begin{tabular}{lcl}
\hline Performance Group & DS & Description \\
\hline PG1 (Pier at base) & DS1 & $\begin{array}{l}\text { Seal cracks and minor re- } \\
\text { moval and patching of con- } \\
\text { crete }\end{array}$ \\
\hline PG2 (Pier at top) & DS0 & ---- \\
\hline PG3 & DS1 & $\begin{array}{l}\text { Seal cracks and minor re- } \\
\text { moval and patching of con- } \\
\text { crete }\end{array}$ \\
\hline PG4 & DS2 & $\begin{array}{l}\text { Seal cracks, clean deck and } \\
\text { apply methacrylate }\end{array}$ \\
\hline
\end{tabular}

Table 2. Damage States (DS) for Minor scenario

\begin{tabular}{lll}
\hline Performance Group & DS & Description \\
\hline PG1 (Pier at base) & DS2 & Seal cracks, major patching \\
\hline PG2 (Pier at top) & DS1 & $\begin{array}{l}\text { Seal cracks and minor re- } \\
\text { moval and patching of con- } \\
\text { crete }\end{array}$ \\
\hline PG3 & DS2 & Replacement of CCB \\
\hline PG4 & DS2 & $\begin{array}{l}\text { Seal cracks, clean deck and } \\
\text { apply methacrylate }\end{array}$ \\
\hline
\end{tabular}

According to the methodology developed in ([3] and [13], once the scenario has been defined its average cost can be calculated by summing the costs of the repairing items associated to each PG. Figure 11 shows the repair-to-rebuild ratio for both minor and major scenarios. Figure 12 shows the cost disaggregated for PGs. From the results it clearly appears that PG1 (base of piers) contributes most to minor damage scenario expected cost at high value of the PGA, while peak contribution is from PG3 (cross beams) at lower values of PGA for the minor scenario and over the whole range of PGA for the major damage scenario. 


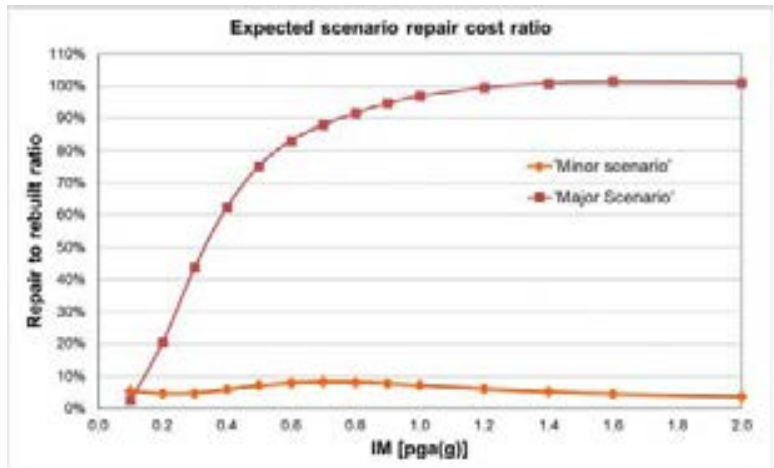

Figure 11. Expected repair cost conditioned to IM for the selected scenarios.

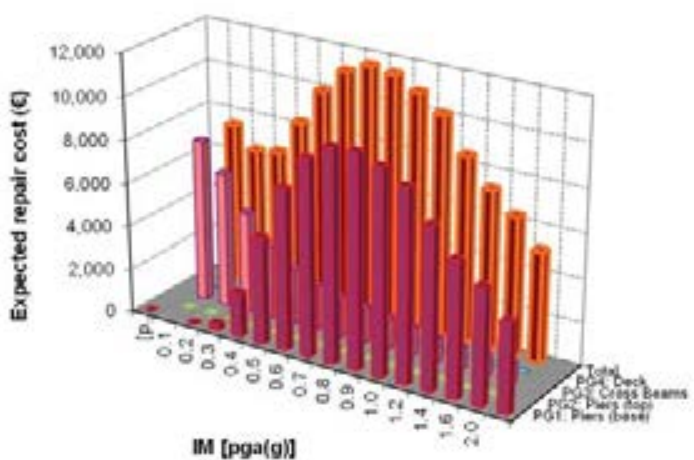

(a)

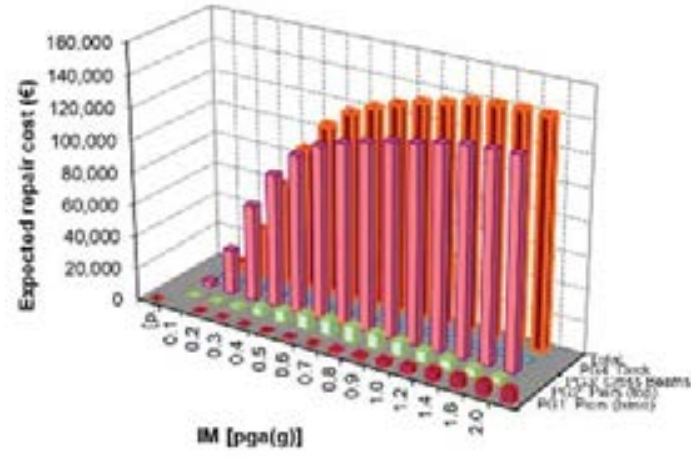

(b)

Figure 12. Disaggregation of the expected (a) minor and (b) major scenario repair cost by PG

From these curves it appears that the contribution for the minor damage scenario is mainly from epoxy inject cracks and repairing minor spalls, independently of earthquake intensity. For major damage scenario temporary support of the deck begins to rise rapidly as a contributing cost for higher intensities. It also can be noted that one repair quantity can dominate because several performance groups require that item. Figure 13 shows the probability of occurrence of the selected scenarios.

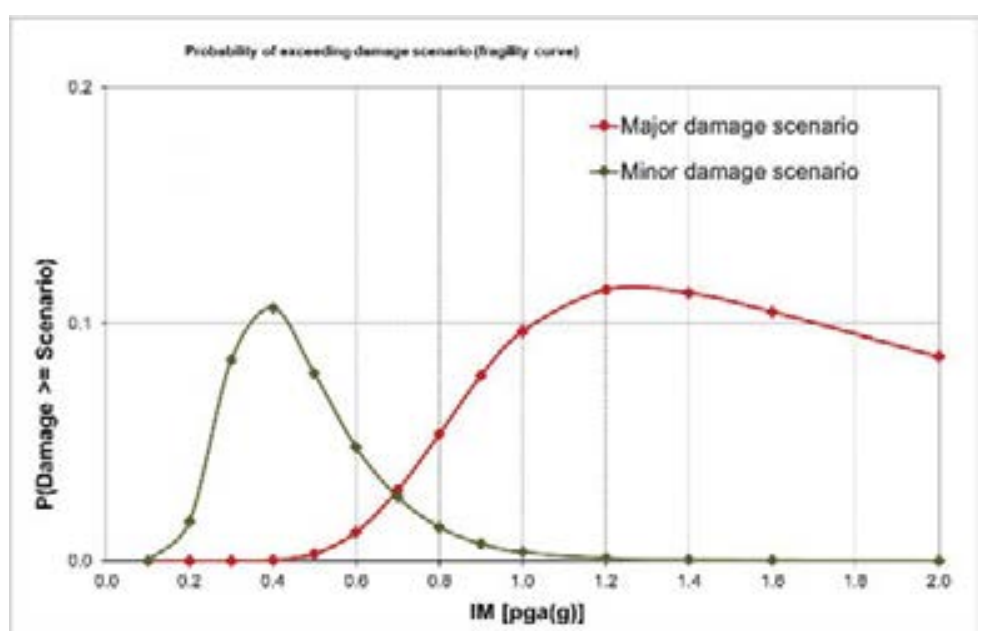

Figure 13. Probability of occurrence of the expected (a) minor and (b) major scenario 
From the application of the methodology to the case study the following observations can be drawn:

- Significant probability of occurrence of the minor damage scenario are due to $0.3 \mathrm{~g}<\mathrm{PGA}$ $<0.6 \mathrm{~g}$;

- Significant probability of occurrence of the major damage scenario are due to PGA $>0.7$ g;

- An overall expected repair ratio between 5-8\% is obtained for the minor damage scenario and $>20 \%$ for PGA $>0.2 \mathrm{~g}$ for major damage scenario;

- PG1, PG2 (pier) and PG3 (CCB) mainly govern the decision-making analysis given that their associated costs results particularly important. Therefore, any optimization action should act on these elements.

\section{CONCLUSIONS}

The SEQBRI project dealt with the full application of the next-generation PerformanceBased Earth-quake Engineering methodology (PBEE) employed for the assessment of a new type of steel concrete composite (SSC) bridges endowed with a special pier-to-deck connection (Concrete Cross Beam).

The development of the PBEE framework for SCC bridges can be considered a novel contribution to the foundation of a new generation of earthquake engineering seismic codes for bridges in Europe. The results are novel. In particular, they entail:

- A better understanding of the seismic behaviour of SCC bridges with CCB;

- A reliable definition of limit states for this new type of bridges

- A proposal of a decision-making tool based on next-generation PBEE methodology for SCC bridges with CCB in moderate/strong seismic prone areas.

- A significant improvement of seismic design state-of-the-art of SCC bridges with CCB, through the development of design guidelines for improving Eurocodes for the assessment of bridges in seismic prone areas.

It is evident that the application of this complex framework can be totally justified only when important damage conditions are expected and for which the adoption of non-linear models and probabilistic analyses appear justifiable. Given the fact that small-medium SCC bridges with CCB exhibit in mean important damage conditions only for PGA $>0.25 \mathrm{~g}$, it appears reasonable to employ the PBEE methodology only for PGA values of $0.35 \mathrm{~g}$ or great-er. These PGA values characterize the most active prone-areas in Europe. For lower PGA values the design approaches to be adopted can rely on current design approaches prescribed by Eurocodes, where partial safety factors cover aleatory uncertainties. In summary, no specific rules are provided by Euro-codes to design CCBs, so, the design procedure pro-posed in the SEQBRI project can be profitable used for this purpose.

\section{AKNOWLEDGMENTS}

The Present work is supported by the funds of the Euro-pean Project SEQBRI: "Performance-Based Earthquake Engineering Analysis of Short-Medium Span Steel-Concrete Composite Bridges", Grant N ${ }^{\circ}$ RFSR-CT-2012-00032. This project has partially received funding from the Italian Ministry of Education, University and Research (MIUR) in the frame of the Departments of Excellence (Grant L. 232/2016). 


\section{REFERENCES}

[1] CEN 1994 2006. "Eurocode 4: Design of composite steel and concrete structures", Brussels, 2006.

[2] Porter, K. A. 2000. An Overview of PEER's Performance-Based Earthquake Engineering Methodology. Ninth International Conference on Applications of Statistics and Probability in Civil Engineering (ICASP9) July 6-9, 2003, San Francisco.

[3] Paolacci, F., et al., 2017. "Performance-based earthquake engineering analysis of short medium span steel-concrete composite bridges", Final Report, SEQBRI Project, Contr. No: RFSR-CT-2012-00032, Research Fund for Coal and Steel. ISBN 978-92-79-656125, ISSN 1831-9424, DOI:10.2777/48012.

[4] Lachal, A, Aribert, J.M. 2002. National program MIKTI, Conception de tabliers performants dans le domaine des petites portées. Sujet 1-B: Techniques innovantes de raboutage, Cahier.

[5] Somja, H., Kaing, S., Lachal, A. 2012. New beam-to-beam joint with concrete embedding for composite bridges Experimental study and finite element modelling Journal of Constructional Steel Research, Vol 77, 210-222

[6] Abbiati G., Cazzador E., Alessandri S., Bursi O.S., Paolacci F., De Santis S. 2018. Experimental characterization and component-based modeling of deck-to-pier connections for composite bridges, Journal of Constructional Steel Research,150, pag. 31-50, DOI: 10.1016/j.jcsr.2018.08.005.

[7] Paolacci F., Giannini R., Alessandri S., Corritore D., (2018), An experimental investigation on the seismic response of a new pier-to-deck connection for composite short-medium span bridges, submitted to Journal of Traffic and Transportation Engineering, Volume 5, Issue 6, December 2018, Pages 439-453, DOI: 10.1016/j.jtte.2018.10.002

[8] Hanswille, G. 2007. "Composite bridges in Germany - State of the Art", Proceedings of 7th German Japanese Bridge Symposium, Osaka.

[9] DIN FB 104 2009, DIN-Fachbericht 104 - Verbundbrücken (Composite steel and concrete bridges). Berlin, 2009

[10] Mazzoni, S., McKenna, F., Scott, M.H., Fenves, G.L. 2009. Open System for Earthquake Engineering Simulation User Command-Language Manual, Pacific Earthquake Engineering Research Center, University of California, Berkeley, May, 2009.

[11] Gattesco, N., Giuriani E. 1996. "Experimental Study on Stud Shear Connectors Subjected to Cyclic Loading," vol. 38, no. 1, pp. 1-21, 1996.

[12] Paolacci, F., Giannini, R., Alessandri, S., Corritore, D. 2018. On the cyclic behaviour of new pier-to-deck connections for short-medium span composite I-girder bridges. Journal of traffic and transportation engineering. 2018; 5(6): 439-453.

[13] CEN 1998-1:2005. Design of Structures for Earthquake Resistance Part 1: General Rules, Seismic Actions and Rules for Buildings. Comitè e Europaèen de Normalisation: Brussels, 2005.

[14] Mackie, K.R., Wong, J.M., Stojadinovic, B. 2008. "Integrated Probabilistic PerformanceBased Evaluation of Benchmark Reinforced Concrete Bridges". Pacific Earthquake Engineering Research Center. 
[15] Tubaldi, E., Barbato, M., and Dall'Asta, A. 2010. "Transverse seismic response of continuous steel-concrete composite bridges exhibiting dual load path". Earthquake and Structures 1:1, 21-41.

[16] Vamvatsikos, D., Cornell, C.A. 2002. Incremental dynamic analysis. Earthquake Engineering and Structural Dynamics 2002; 31:491-514.

[17] FEMA 356 2000: Prestandard and Commentary for the Seismic Rehabilitation of Buildings, Prepared by American Society of Civil Engineers, Washington, D.C. (U.S.A.), November.

[18] Berry, M.P., Eberhard, M. 2003. "Performance models for flexural damage in reinforced concrete columns". PEER Report, 2003/18.

[19] Alessandri, S., Giannini, R. and Paolacci, F. 2013. Aftershock risk assessment and the decision to open traffic on bridges, Earthquake Engng. Struct. Dyn., 42: 2255-2275. 